\title{
Editor's choice to the October 2021 issue
}

\author{
Johan H. C. Reiber ${ }^{1}$
}

Published online: 17 September 2021

(C) The Author(s), under exclusive licence to Springer Nature B.V. 2021

Dear reader,

For this October 2021 issue, I have selected and would like to recommend a review paper entitled "Role of cardiovascular magnetic resonance in early detection and treatment of cardiac dysfunction in oncology patients" by Dr Srilakshmi Vallabhaneni and co-authors form the Washington University School of Medicine, St Louis, USA and the Department of Cardiology, University Hospital Heidelberg in Germany [1]. They clearly describe how cardiovascular magnetic resonance imaging is able to identify subclinical cancer therapy related cardiac dysfunction in patients receiving potentially cardiotoxic chemotherapy and to guide initiation of cardio protective therapy. This paper is also illustrated with case examples, demonstrating how multi-parametric analysis with myocardial strain, and tissue characterization, such as T1 and T2 mapping as well as late gadolinium enhancement, play a critical role in understanding important clinical questions in cardio-oncology.

This one example is their Fig. 1, that illustrates the improvement in LV function of a patient after 12 months FU, who was on a cardiac protection and exercise regimen. But there are also other nice illustrations using tissue characterization.

I wish you much pleasure in reading this and other papers in this issue of the International Journal of Cardiovascular Imaging.
Johan H. C. Reiber

J.H.C.Reiber@lumc.nl

1 Leiden University Medical Center, Leiden, The Netherlands 
Fig. 1 Long axis SSFP images of case 1. Baseline CMR at presentation with reduction in left ventricular ejection fraction (LVEF) at 34\% with increased left ventricular end-diastolic (LVEDV) (a) and end-systolic (LVESV) (b) volumes along with mild to moderate mitral regurgitation (yellow arrow). On cardio-protection and exercise regimen, 12 month follow-up showing improvement in LVEF to $51 \%$ with improved LVEDV (c) and LVESV (d) along with improvement in mitral regurgitation (red arrow)
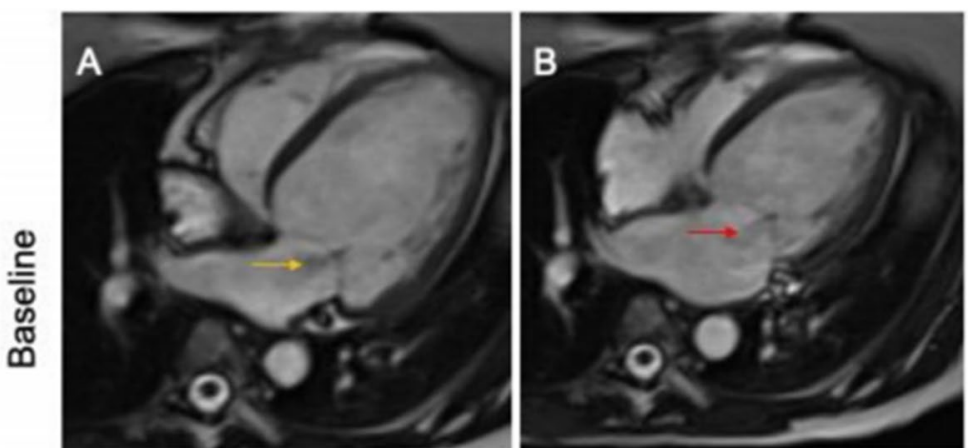

LVEF- $34 \%$

LVEDV- $211 \mathrm{ml}$

LVESV- $140 \mathrm{ml}$

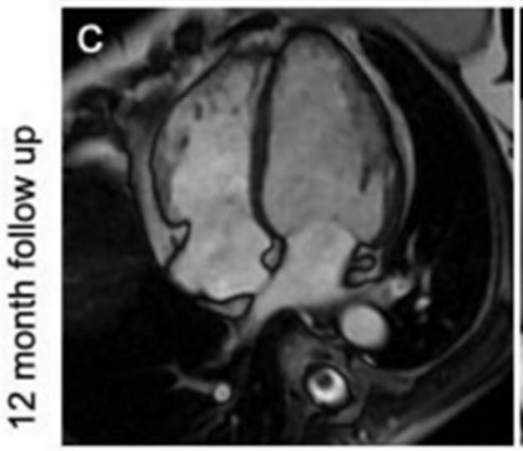

LVEF- $51 \%$

LVEDV- $125 \mathrm{ml}$

LVESV- $62 \mathrm{ml}$

\section{Declarations}

Conflict of interest The author declare that they have no conflict of interest.

\section{Reference}

1. Vallabhaneni S, Zhang KW, Alvarez-Cardona JA et al (2021) Role of cardiovascular magnetic resonance in early detection and treatment of cardiac dysfunction in oncology patients. Int J Cardiovasc Imaging. https://doi.org/10.1007/s10554-021-02271-7

Publisher's Note Springer Nature remains neutral with regard to jurisdictional claims in published maps and institutional affiliations. 